$R R-74-24$

A NEW EQUATION FOR THE LINEAR REGULATOR PROBLEM

J. Casti

December 1974

Research Reports are publications reporting on the work of the author. Any views or conclusions are those of the author, and do not necessarily reflect those of IIASA. 



\section{A New Equation for the Linear}

Regulator Problem

J. Casti*

\section{Abstract}

In this article a new equation is derived for the optimal feedback gain matrix characterizing the solution of the standard linear regulator problem. It will be seen that, in contrast to the usual algebraic Riccati equation which requires the solution of $n(n+1) / 2$ quadratically nonlinear algebraic equations, the new equation requires the solution of only $\mathrm{nm}$ such equations, where $m$ is the number of system input terminals, and $\mathrm{n}$ is the dimension of the state vector of the system.

Utilizing the new equation, results are presented for the inverse problem of linear control theory.

\section{Introduction}

We consider the problem of minimizing

$$
J=\int_{0}^{\infty}[(x, Q x)+(u, u)] d t
$$

over all piecewise continuous control laws $u(t)$, where the state $x$ and the control $\mathbf{u}$ are connected by the linear differential equation

$$
\frac{d x}{d t}=F x+G u, \quad x(0)=c \text {. }
$$

Here $x$ and $u$ are $n, m-d i m e n s i o n a l$ vectors, respectively, $F, Q$, and $G$ are $n \times n, n \times n$, and $n \times m$ constant matrices, with $Q$ non-negative definite. Well known [1] results from optimal control theory state that the solution to this problem is

$$
\begin{aligned}
& J_{\min }=(c, P c), \\
& U_{\min }(t)=-G^{\prime} P_{x}(t),
\end{aligned}
$$

\footnotetext{
*International Institute for Applied Systems Analysis, Laxenburg
} Austria. 
where $P$ is the unique non-negative definite solution of the algebraic Riccati equation

$$
Q+P F+F^{\prime} P-P G G^{\prime} P=0 .
$$

The quantity $H^{\prime}=G^{\prime} P$ is, for obvious reasons, called the optimal feedback gain matrix and, if the pair $(F, G)$ is controllable while the pair $\left(F, Q^{\frac{1}{2}}\right)$ is observable, then it can be shown that the closed loop system

$$
\frac{d x}{d t}=\left(F-G G^{\prime} P\right) x
$$

is also asymptotically stable.

The inverse problem of linear control theory is the following: Given an asymptotically stable control law G'P, characterize all positive semi-definite matrices $Q$ leading to the given control law through the ARE.

Notice that the ARE represents a set of $n(n+1) / 2$ quadratically nonlinear algebraic equations for the components of $P$ as $P$ is symmetric. Various successive approximation $[10]$ and direct $[11]$ methods have been proposed for finding the positive semi-definite solution of ARE.

In this paper, we shall present a new equation which is also quadratically nonlinear but in the components of $H$ and not $P$. Since $H$ is an nxm matrix, the new equation represents only $\mathrm{nm}$ algebraic equations which, if $m<(n+1) / 2$, represent fewer equations than those needed for the ARE. Also, it will be seen that the new equation has some analytic features which enable us to easily obtain some useful results relating to the inverse problem.

\section{Main Results}

Before proceeding to the principal results, recall a few useful facts from matrix theory: let $\mathrm{A}$ and $\mathrm{B}$ be $\mathrm{nxm}$, pxq matrices, respective1y. Then the Kronecker product of $A$ and $B$, denoted $A \otimes B$, is the npxmq matrix whose $(i, j)^{\text {th }}$ element is $a_{i j} B, i=1, \ldots, n p, j=1,2, \ldots m q$. 
Next, let $A$ be an $n x m$ matrix. By $\sigma(A)$ we denote the nmxl matrix formed from A by "stacking" the columns of A beginning with the first. Thus,

$$
\sigma(A)=\left(a_{11} a_{21} \cdots a_{n 1} a_{12} a_{22} \cdots a_{n 2} \cdots a_{n n}\right)^{\prime} \cdot
$$

In terms of the operations $\otimes$ and $\sigma$, we can obtain the useful formula [2]

$$
\sigma(\mathrm{PAQ})=\left(\mathrm{Q}^{\prime} \otimes \mathrm{P}\right) \sigma(\mathrm{A}),
$$

valid for any three matrices $P, A, Q$ for which the product $P A Q$ makes sense. This result will be exploited below. Note also that $\sigma(A+B)$ $=\sigma(A)+\sigma(B)$ and $\sigma(\alpha A)=\alpha \sigma(A)$ for any scalar $\alpha$, so that $\sigma$ is a linear operator from the space of mxn real (or complex) matrices to $\mathrm{R}^{\mathrm{nm}}$ (or $\mathrm{C}^{\mathrm{nm}}$ ). We use these facts now to prove our

Main Theorem. Assume the system matrix $F$ has no characteristic roots on the imaginary axis. Then the optimal feedback gain matrix $\mathrm{H}$ satisfies the equation.

$$
\sigma(H)=\left(G^{\prime} \otimes I\right)\left(I \otimes F^{\prime}+F^{\prime} \otimes I\right)^{-1} \sigma\left(H H^{\prime}-Q\right) .
$$

Proof. BY definition $H=P G$. Thus, the ARE may be written as

$$
Q+P F+F^{\prime} P-H H^{\prime}=0 .
$$

Applying the operation $\sigma$ and relation (1) we obtain

$$
\left(I \otimes F^{\prime}+F^{\prime} \otimes I\right) \sigma(P)=\sigma\left(H H^{\prime}-Q\right) .
$$

The assumption on $F$ insures that $\left(F \otimes F^{\prime}+F \otimes I\right)$ is non-singular. Thus,

$$
\sigma(P)=\left(I \otimes F^{\prime}+F^{\prime} \otimes I\right)^{-1} \sigma\left(H H^{\prime}-Q\right)
$$

However, $\sigma(H)=\sigma(P G)=\left(G^{\prime} \otimes I\right) \sigma(P)$. Thus multiplying (2) by $\left(G^{\prime} \otimes I\right)$, we obtain (HE).

Remarks. i) As noted above, HE represents nm quadratically 
nonlinear algebraic equations for the components of $\mathrm{H}$. Thus, the "algebraic degree" of difficulty in solving $\mathrm{HE}$ is the same as that in solving the ARE. However, if $m<(n+1) / 2$, there are fewer equations in $\mathrm{HE}$ and so it would appear the computing burden is reduced. More discussion of this matter is given in section III.

ii) From the point of view of applying various successive approximation methods, $\mathrm{HE}$ is in better form than ARE since the unknown quantity $\mathrm{H}$ stands alone on the left side of HE. This enables standard algorithms to be applied without further manipulation.

iii) The solution of $\mathrm{HE}$ is the limiting function for the generalized $X-Y$ equations introduced in $[3,4,5,9]$. It is interesting to observe that HE cannot be obtained by a simple examination of the critical points of. the finite interval cauchey problem, but must be approached through the procedure employed above.

iv) It is to be expected that all properties of the ARE such as uniqueness of the positive, semi-definite solution, monotonicity of approximants in numerical schemes, etc. may be extended to the HE, the appropriate definitions and interpretations being induced by the definition $\mathrm{H}=\mathrm{PG}$, e.g. the natural ordering $\mathrm{H}_{1} \geq \mathrm{H}_{2}$ would follow from $\mathrm{P}_{1}$ $\geq \mathrm{P}_{2}$, where $\mathrm{H}_{1}=\mathrm{P}_{1} \mathrm{G}_{2} \mathrm{H}_{2}=\mathrm{P}_{2} \mathrm{G}$. We now employ the $\mathrm{HE}$ in order to prove an important result for the inverse problem of linear control theory.

Inverse Problem Theorem. Let $(F, G)$ be controllable and let $H_{1} H_{2}$ be ti.e solutions of the equations

$$
\begin{aligned}
& \sigma\left(\mathrm{H}_{1}\right)=\left(\mathrm{G}^{\prime} \otimes \mathrm{I}\right)\left(\mathrm{I \otimes F^{ \prime }}+\mathrm{F}^{\prime} \otimes \mathrm{I}\right)^{-1} \sigma\left(\mathrm{H}_{1} \mathrm{H}_{1}^{\prime}-\Omega_{1}\right), \\
& \sigma\left(\mathrm{H}_{2}\right)=\left(\mathrm{G}^{\prime} \otimes \mathrm{I}\right)\left(\mathrm{I \otimes F^{ \prime }}+\mathrm{F}^{\prime} \otimes \mathrm{I}\right)^{-1} \sigma\left(\mathrm{H}_{2} \mathrm{H}_{2}^{\prime}-\mathrm{Q}_{2}\right),
\end{aligned}
$$

$\because \ldots$ E $Q_{1}, Q_{2}$ are positive semi-definite, $\left(F, Q_{1}^{\frac{1}{2}}\right),\left(F, Q_{2}^{\frac{3}{2}}\right)$ being observable. Then a necessary and sufficient condition for $(*)$ and (**) to I. Ive the same positive semi-definite (see note iv above) solution is 


$$
\sigma\left(Q_{1}-Q_{2}\right) \varepsilon \operatorname{ker}\left[\left(G^{\prime} \otimes I\right)\left(I \otimes F^{\prime}+F^{\prime} \otimes I\right)^{-1}\right] .
$$

Proof. We first remark that the controllability, observability, and definiteness assumptions imply, through standard arguments and the definition $H=P G$, that both $\left(^{*}\right)$ and $\left({ }^{*}\right)$ have a unique, positive, semidefinite solution. We denote this solution by $\mathrm{H}_{1}^{+}, \mathrm{H}_{2}^{+}$, respectively. We first prove necessity.

Let $\mathrm{H}_{1}^{+}=\mathrm{H}_{2}^{+}$. Subtracting $(* *)$ from $(*)$, we have

$$
\begin{aligned}
& 0=\sigma\left(\mathrm{H}_{1}^{+}\right)-\sigma\left(\mathrm{H}_{2}^{+}\right)=\left(\mathrm{G}^{\prime} \otimes \mathrm{I}\right)\left(\mathrm{I} \otimes \mathrm{F}^{\prime}+\mathrm{F}^{\prime} \otimes \mathrm{I}\right)^{-1} \\
& \times\left[\sigma\left(\mathrm{H}_{1}^{+} \mathrm{H}_{1}^{+}\right)-\sigma\left(\mathrm{H}_{2}^{+} \mathrm{H}_{2}^{+}\right)+\sigma\left(\mathrm{Q}_{2}\right)-\sigma\left(Q_{1}\right)\right] .
\end{aligned}
$$

Thus, $\sigma\left(Q_{2}-\Omega_{1}\right) \varepsilon \operatorname{ker}\left[\left(G^{\prime} \otimes I\right)\left(I \otimes F^{\prime}+F^{\prime} \otimes I\right)^{-1}\right]$.

on the other hand, let the condition be satisfied. We write $\left(^{*}\right)$ and $(* \star)$ as

$$
\begin{aligned}
& \left(G^{\prime} \otimes I\right)\left(I \otimes F^{\prime}+F^{\prime} \otimes I\right)^{-1} \sigma\left(H_{1} H_{1}^{\prime}\right)-\sigma\left(H_{1}\right) \\
& =\left(G^{\prime} \otimes I\right)\left(I \otimes F^{\prime}+F^{\prime} \otimes I\right)^{-1} \sigma\left(Q_{1}\right), \\
& \left(G^{\prime} \otimes I\right)\left(I \otimes F^{\prime}+F^{\prime} \otimes I\right)^{-1} \sigma\left(H_{2} H_{2}^{\prime}\right)-\sigma\left(H_{2}\right) \\
& =\left(G^{\prime} \otimes I\right)\left(I \otimes F^{\prime}+F^{\prime} \otimes I\right)^{-1} \sigma\left(Q_{2}\right) .
\end{aligned}
$$

Thus, since the right-hand sides of $(*)$ and $(* *)$ are the same, we see that $\mathrm{H}_{1}$ and $\mathrm{H}_{2}$ both satisfy the same equation and, since this equation has a unique positive semi-definite solution, the theorem is proved.

Remarks. i) Since $\sigma\left(Q_{2}-Q_{1}\right)$ contains $n^{2}$ elements, while $\operatorname{ker}\left[\left(G^{\prime} \otimes I\right)\left(I \otimes F^{\prime}+F^{\prime} \otimes I\right)^{-1}\right]$ is of dimension $n m$, there are $n(n-m)$ degrees of freedom in selecting an element $Q_{2}-\Omega_{1} \varepsilon$ ker [:]. However, the semi-definiteness and controllability requirements may reduce this to a lesser number, depending upon the structure of $F$. 
ii) The foregoing result easily enables us to characterize all $Q_{2}$ which are equivalent to a fixed $Q_{1}$, in the sense of generating the same feedback law. Explicitly, for all such $Q_{2}$ we have

$$
\sigma\left(Q_{2}\right)=\sigma\left(Q_{1}\right) \oplus \operatorname{ker}\left[\left(G^{\prime} \otimes I\right)\left(I \otimes F^{\prime}+F^{\prime} \otimes I\right)^{-1}\right] \text {, }
$$

when $\oplus$ denotes the direct sum of two vector spaces.

\section{Discussion}

In this paper, we have presented a new equation characterizing the optimal linear regulator gain matrix and have used this equation to obtain some new results for the inverse problem of 1 inear control theory. Several topics associated with these results are currently under investigation and will be reported on in future publications. Among these are:

a) Computational procedures - even though HE usually results in a fewer number of equations to solve than ARE, the presence of the term $\left(I \otimes F^{\prime}+F^{\prime} \otimes I\right)^{-1}$, requiring the inverse of an $n^{2} \times n^{2}$ matrix, introduces some nontrivial computational considerations. By various partitioning methods this inverse may be calculated in $O\left(n^{4}\right)$ multiplications, but it is not yet clear that solving HE always results in a faster computation than solving ARE. Also, comparative investigations of direct vs. iterative methods need to be carried out, as well as studies for the case when $F$ has multiple characteristic roots;

b) Analytical Studies - the equation HE is a substantial generalization of the H-equation of radiative transfer, which was originally studied by Ambartsumian and Chandrasekhar. Several interesting properties of the $H$ function have been obtained in the radiative transfer case, and it is now a matter of some interest to see if generalizations may be obtained. From the results of $[7,12]$, it would appear promising, but the details remain to be worked out.

Also, it is well-known that there exist intimate connections 
between the Riccati equation and linear differential systems. It would be interesting to know if the solution of the HE also has such connections, particularly since one of the primary methods for solving the ARE is based upon this connection [II];

c) Infinite-Dimensional Problems - the infinite-dimensional, finite time, quadratic cost-linear dynamics control problem was studied in [6] from the point of view of obtaining alternates to the operator Riccati equation for characterizing the optimal feedback law. It was shown that whenever there are only a finite number of places where control may be exerted or measurements made, the new equation resulted in a computational improvement over the operator Riccati equation. It seems natural to conjecture that the HE may be extended to the operator situation with no substantial difficulty but again the details are still under investigation. 


\section{References}

[1] Barnett, S. Matrices in Control Theory, London, Van Nostrand Reinhold, Co., 1971.

[2] Bellman, R. Introduction to Matrix Analysis, 2nd ed., New York, McGraw-Hill Co., 1970.

[3] Casti, J. "Matrix Riccati Equations, Dimensionality Reduction, and Generalized X-Y Functions," Utilitas Mathematics (to appear November 1974).

[4] Casti, J. "Reduction of Dimensionality for Systems of Linear Two-Point Boundary Value Problems with Constant Coefficients," J. Math. Analy. Applic., 45 (1974), 522-531.

[5] Casti, J. R. Kalaba, and K. Murthy. "A New Initial Valve Method for on-line Filtering and Estimation," IEEE Trans. Info. Theory, IT-18 (1972), 515-518.

[6] Casti, J. and L. Ljung. "Some New Analytic and Computational Results for Operator Riccati Equations" (to appear in SIAM J. Control, 1974).

[7] Casti, J. and E. Tse. "Optimal Linear Filtering Theory c.nd Radiative Transfer: Comparisons and Interconnections," J. Math. Analy. Applic., 40 (1972), 45-54 .

[8] Chandrasekhar, S. Radiative Transfer, New York, Dover Publ. Co., 1960 .

[9] Kailath, T. "Some Chandrasekhar-Type Algorithms for Quadratic Regulators," Proc. IEEE Dec. \& Control Conf., New Orleans, December 1972 .

[10] Kleinman, D.L. "On an Iterative Technique for Riccati Equation Computations," IEEE Trans. Auto Control, AC-13 (1968), 114-115.

[11] Potter, J.E. "Matrix Quadratic Solutions," SIAM J.Appl. Math., $14(1956), 496-501$.

[12] Sidhu, G. and J. Casti. "Contributions to a Unification of the Theories of Radiative Transfer and Linear Stochastic Filtering" (to appear in SIAM Review, 1974). 